\title{
Learning Curve Associated with Complications in Biportal Endoscopic Spinal Surgery: Challenges and Strategies
}

\author{
Dae-Jung Choi, Chang-Myong Choi, Je-Tea Jung, Sang-Jin Lee, Yong-Sang Kim \\ Spine Center, Barun Hospital, Jinju, Korea
}

Study Design: Descriptions of technical strategies to overcome pitfalls associated with early learning periods in biportal endoscopic spinal surgery (BESS).

Purpose: To introduce BESS for lumbar spinal diseases (LSDs) and to inform certain challenges to be overcome in mastering the technique.

Overview of Literature: BESS has shown superior benefits including excellent magnification, a wider range of view by dynamic handling of an endoscope and instruments. Clinical reports, however, have not yet been very revealing for its new introduction into minimally invasive spine surgery.

Methods: To evaluate the learning curve for BESS, the procedures for various LSDs by one surgeon were analyzed in the view of shortening of the operating times and reduction of complications. Reviewing of recorded procedures helped in finding the reasons and the implemented solutions.

Results: The 68 cases included 25 for lumbar disc herniation (LDH), 3 for revision for recurred LDH, 39 for lumbar spinal stenosis (LSS) and 1 for synovial cyst. The operation time for the total cases averaged 83.7 \pm 33.6 minutes. According to diagnosis, it was $68.2 \pm 23.7$ minutes for LDH. After the 14th case of LDH, it was nearly constant and close to the average time. One level of LSS needed $110.4 \pm 34.4$ minutes. Prolonged operation times even in some later cases of LSS were mainly from struggling against blurred vision due to epidural bleeding. There were 7 cases of complications (10.3\%) including 2 cases of dural tear, 1 case of root injury, and 4 cases of incomplete decompression on postoperative magnetic resonance imaging. There was no case of symptomatic hematoma or wound infection.

Conclusions: BESS seemed to have a relatively short learning curve period. The overall complication rate in early learning period was $10.3 \%$. These could be avoided by magnified regional views on an endoscope and a clear surgical field by controlling epidural bleeding.

Keywords: Lumbosacral; Spinal stenosis; Complication; Minimally invasive surgical procedure; Endoscopic

\section{Introduction}

There have been several trials of minimally endoscopic spine surgery to treat lumbar spine diseases [1,2]; however, technical barriers including stiff handling of an endoscope and limitation of instruments available could

Received Oct 11, 2015; Accepted Nov 11, 2015

Corresponding author: Dae-Jung Choi

Spine Center, Barun Hospital, 7 Dongjin-ro, Jinju 52725, Korea

Tel: +82-55-790-3000, Fax: +82-55-790-3030, E-mail: djchoi9@hanmail.net 
only be undertaken by experienced endoscopic surgeons [3]. There has been the recent introduction of biportal endoscopic spinal surgery (BESS) to minimally invasive spine surgery (MISS) as it has several benefits including excellent magnification and illumination just as for conventional spinal endoscopy through one portal. In addition, a wider range of view by a freely moving endoscope and dynamic handling of various instruments permits in overcoming the limitation of surgical indication for conventional spinal endoscopy (Fig. 1).

In the early learning periods for this technique, however, there needs to be a discussion of various endoscopic circumstances and challenges. The authors discuss the complications associated with BESS for surgeons new to the technique and strategies to overcome them.

\section{Materials and Methods}

Consecutive 146 cases of BESS were performed for treating of various spinal diseases from January to May of 2015 in one spine center. To evaluate the learning curve, 68 cases of a certain surgeon were selected and analyzed in view of shortened operation times and reduction in the complication rates. The surgeon had 8 years of experience in spine surgery, but had started performing endoscopic spinal surgery by himself.

Operating times were analyzed in view of the diagnosis. The time by which performances had gone smoothly and as planned was assumed to be the endpoint of the learning curve for the technique. Technical complications on recorded performances were reviewed to find the causes

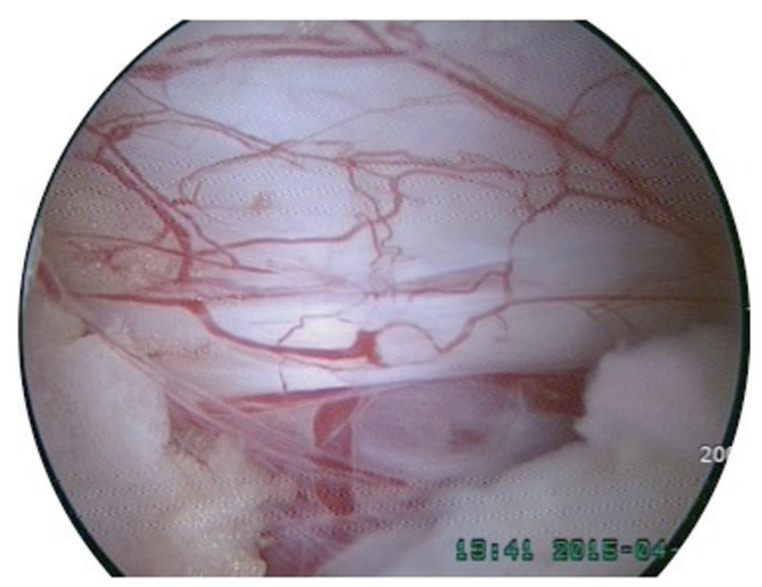

Fig. 1. An endoscopic view. The magnified view showed very small epidural vessels clearly. and discover strategies to address them.

\section{Surgical technique of BESS}

A patient was placed in the prone position over the radiolucent chest frame in a flexed position. Fluoroscopic confirmation of a certain level had to be made before incision. Two standard entry points were going to be made at 1 $\mathrm{cm}$ above and below the disc space on an anteroposterior view. But skin markings of entry points before incision under fluoroscope was sometimes to the wrong level due to the lumbar curvature and the depth of back muscle. Confirming the level, therefore, was necessary after placing a freer just on the targeted facet joint on a lateral view. Some parts of muscles just medial to the side of the facet joint were split with a blunt dilator, followed by an endoscope and a freer touching on the targeted facet joint. Muscle detachment with the dilator on the distal margin of the lamina and medial side of the facet joint before inserting an endoscope was necessary to prepare enough visual space for allowing to work in earnest. The tip of an endoscope needed to be placed between just proximal of the distal margin of the lamina to the top of the facet joint on the left side approach and just distal to the distal margin on the right side approach. Such positioning would help handling of instruments easier if the operator is right-handed.

Fluent water flow over the inlets should be checked for not increasing the water pressure in the spinal canal and over compressing the dura that might lead to an increased intracranial pressure. Natural pressure from a 3,000 mL of saline bag at 50 to $80 \mathrm{~cm}$ higher than the level of the patient's back could be sustained under this flow. If the flow was stopped after inserting of instruments particularly under thicker back muscles, punching-out or a little cross cut of muscle fascia under the skin could be helpful. If the fluent water outflow were not secured, the back muscle would look swollen and visual field blurred by the bloodtinged turbid water.

After keeping water flow fluent and catching the facet joint in front of the visual field, the next procedure could proceed just as is done in open surgery using high-speed burrs and Kerrison punches. Angled curettes must surely be useful during probing between structures and performing flavectomy (Fig. 2). To approach the contralateral side to decompress both sides in lumbar spinal stenosis (LSS), securing of working space enough for an endoscope and a 
certain working instrument should be prepared with partial resection of the ends of the spinous processes. During flavectomy of the contralateral side, try first to detach or loosen the ligamentum flavum from the margin of medial and dorsal sides of contralateral facet joint just as it was done on the ipsilateral side. Going forward with pushing an instrument under the tight ligamentum flavum could savor the dura or a traversing root on the contralateral side. Use an angled curette with smaller head size (\#0/2 or $\# 0 / 3$ ) or a freer with the smooth head for probing between the dura and ligamentum flavum. Generally three roots of the four in one segment could be observed well. But to observe an exiting root of the ipsilateral side, the inlet for an endoscope would be better start somewhat lower and medial to the side and somewhat higher laminectomy would also be needed. The contralateral approach might

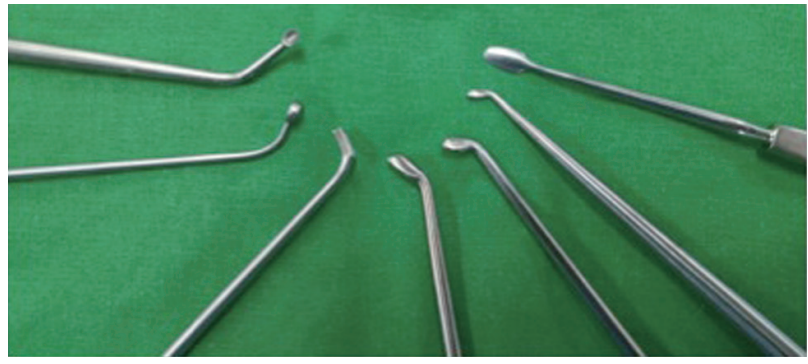

Fig. 2. The useful instruments for biportal endoscopic spinal surgery. Angled instruments were very helpful. These instruments had small and smooth heads.
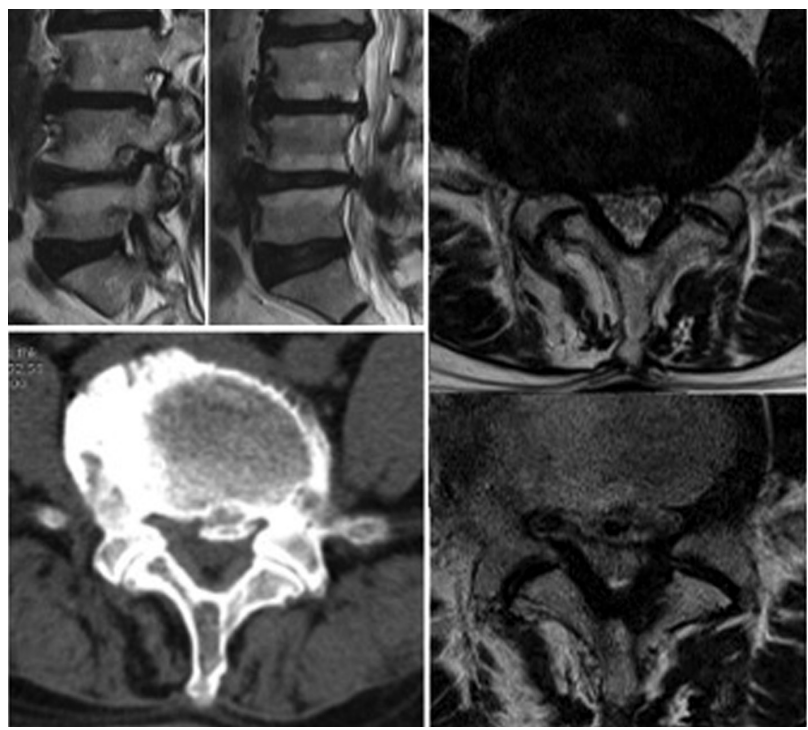

Fig. 3. Preoperative radiographics on the lateral recess stenosis and foraminal stenosis on the right side with calcified lumbar disc herniation in a 58-year old, male patient. be easier to explore and to check out lesions in foraminal stenosis or for far lateral lumbar disc herniation (LDH) in some cases (Figs. 3, 4). Muscle bleeding was controlled by radiofrequency ablation. Bone bleeding as coming out from the laminectomized bone edge was compressed by squashing a piece of bonewax on the bleeding foci. Epidural bleeding just after flavectomy from epidural small vessels around the distal and proximal laminectomized bone could be compressed with a piece of gelfoam pushed into the space between the dura and the lamina followed by rocking a lump of bonewax on it (Push-Rock method), which prevented the gelfoam from coming back out by dural pulsation (Fig. 5). Packing of a bit of bone wax on the bleeding focus under the lamina without a piece of gelfoam could not sufficiently block epidural bleeding, which could turn around and come out the other end.

\section{Results}

The 68 cases were with 28 males, and 40 females; the average age was $58.0 \pm 15.3$ years (range, $23-85$ years). The cases included 25 of LDH (one-level 23, two-level 2), 3 of revision LDH, 39 of LSS (stenosis 28, stenosis with LDH 11) and 1 of synovial cyst.
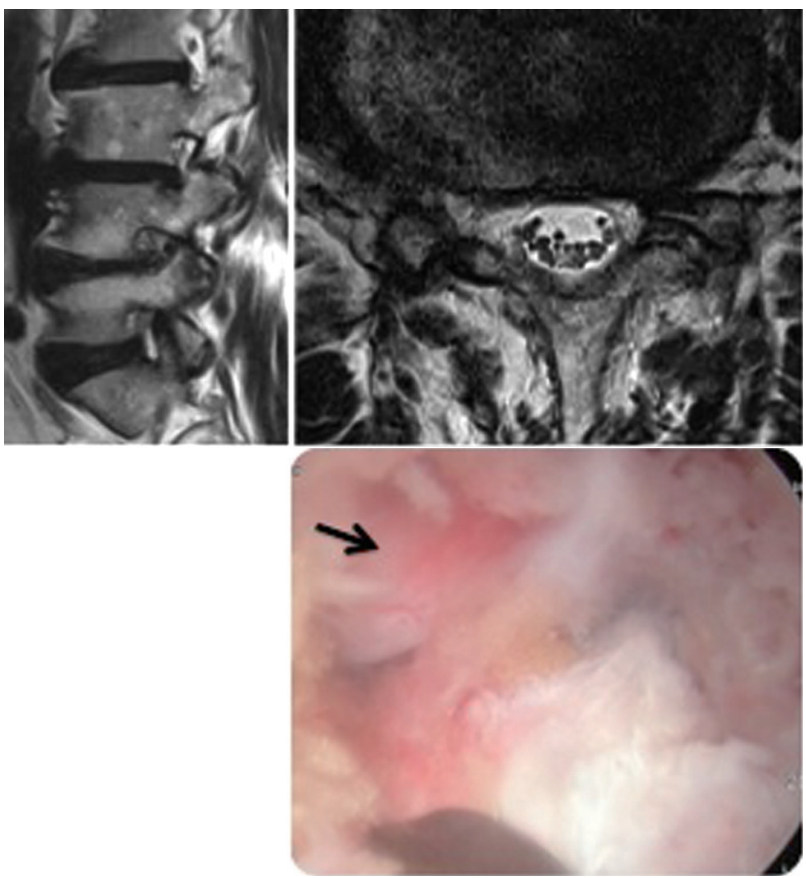

Fig. 4. Postoperative radiographics showed that both lateral recesses and right-sided foramen were decompressed well. An arrowed on endoscopic view indicated an exiting root at the level of the 4-5th lumbar spines. 


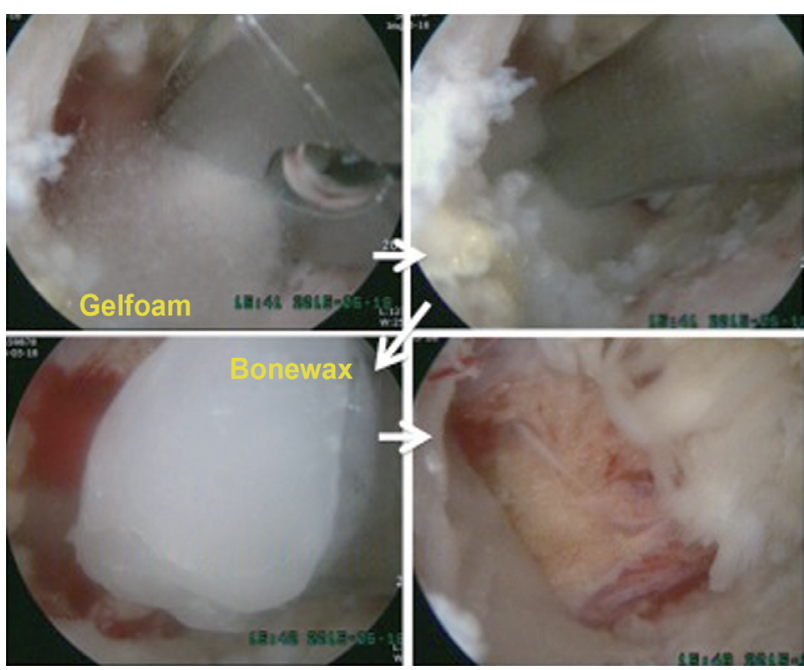

Fig. 5. The Push-Rock method. A piece of gelfoam was inserted and packed under the lamina with a freer. A lump of bonewax was then rocked on it and smashed to compress the bleeding site.

Average operation time for one level of performance was $83.7 \pm 33.6$ minutes, and two levels was $128.6 \pm 14.7$ minutes. For one level of $\mathrm{LDH}$, it was $68.2 \pm 23.7$ minutes. After the 14th case in LDH (the 36th case in total), it remained constant nearly at the average. The operation time for one level of LSS was $110.4 \pm 34.4$ minutes. Prolonged operation times even in later cases of LSS were mainly due to blurred vision by epidural bleeding.

Overall complications during the early learning period included 2 cases of dural tear at the third and 11th cases, root injury at the fifth case, 4 cases of incomplete decompression in LSS, which were revealed on postoperative magnetic resonance imaging (MRI) at the second, 11th, 18 th and 20th cases. There were no instances of symptomatic hematoma or wound infection.

\section{Discussion}

For decades, widely open decompression has been thought to be the gold standard treatment for LSS. But extensive soft tissue dissection caused paravertebral muscles atrophy and might result in back heaviness and long-term pain [4]. Resection of interspinous ligament for better surgical vision also led to instability, and it would require instrumentation $[5,6]$. In recent trials, less invasive approaches have been introduced that have reduced negative hazard and permit accessibility for bilateral decompression. For example, there is microendoscopic surgery through a tubular retractor with paravertebral or midline approach and modified spinous process osteotomy [79]. But MISS using a tubular retractor means a narrow pathway with a small opening through which your visual range could be limited and your handling of instruments might be also restricted. Surgical treatment for various lumbar spine diseases should focus on "reducing sacrifice of the negative components" including skin, paravertebral muscles, and interspinous ligaments, while at the same time attempting surgical removal of the causative structures of hypertrophied ligamentum flavum and outgrowing spur of superior articular processes. With this thinking in mind, endoscopic trials have increased in recent years, and this has led to benefits including minimizing blood loss, shortening operating time, reducing complications and early discharge [10]. Technically, however, endoscopic methods have seemed to be technically challenging and have only covered a limited number of indications. The conventional endoscopic spine surgery through one portal was limitedly recommended only for experienced endoscopic surgeons and large sized central LDH was not recommended according to the International Society for Minimal Intervention in Spinal Surgery guidelines (www. ismiss.com) [3].

BESS has changed the concept of using two portals rather than one portal. One of them is for an endoscope for viewing and the other for certain instruments for the operation. That is a big improvement over the one-portal method, allowing a wider range of view with free handling of basic instruments available in open spine surgery, which can make it possible to decompress a stenotic lesion wider and more safely, while preserving the paravertebral muscles and interspinous ligaments for posterior integrity of the spinal column. If one is experienced with open spine surgery and is familiar with the inner structures and the possible pathologic lesions, overall technical performance may not be difficult. But for a novice, initially making visual space by detachment of some of muscles at the working basement, keeping fluent saline outflow, controlling epidural bleeding, and making a careful approach to the contralateral side may be challenging and may require practice.

The overall complication rate in BESS was about $10.3 \%$. Of these, dura, root injury and blurred vision by epidural bleeding are thought to be from operator being new to the surgical technique. The surgeon may not be accustomed to the new circumstances including the distance, depth and size of the magnified structures, and these may not be 
differentiated in an obscure field in turbid water tinged by epidural bleeding. To keep the surgical field clear, fluent water outflow would be helpful in flushing out the turbid water; in addition, bleeding control from edged bone or epidural small vessels could be done with the Push-Rock method. If the bleeding cannot be controlled with every effort, lowering of diastolic blood pressure to $90-100 \mathrm{~mm}$ $\mathrm{Hg}$ might be helpful in some cases. But increasing the saline pressure by raising the saline bag or squeezing the saline bag is not recommended. That could lead to increased intracranial pressure in the patient and might sometimes cause headache after recovery from anesthesia or cause a delayed recovery from general anesthesia with stiff posture or hyperventilation. For management of a dura tear in BESS, there has not yet been a recommendable technique. But a few pieces of gelfoam laid on the site piece by piece may be remedy, as dural repair is yet impossible with the current instruments. For the two cases of dural tear, there was no cystic or fluid collection seen on postoperative MRIs. That may have been due to the preservation of heavy back muscles overlying the facet joints by the endoscopic approach and blood clot compressing over the gelfoam giving little third space. Trial of suture of the dura with a small needle might be recommendable, but somewhat dangerous if the needle is flushed into epidural or intradural space by the saline flow. If it is needed, conversion to an open procedure is thought to be safer and recommendable.

For the early cases, postoperative MRI revealed that proximal and contralateral sides of ligamentum flavum were not sufficiently removed. Those patients showed resolving of acute neurologic symptoms but continuously complained of fatigue of the lower leg on the concerned side. To perform flavectomy sufficiently, from personal experience, angled curettes have been more helpful than Kerrison punches. Scraping the ligamentum flavum under the lamina could be possible by angled curettes without too much higher laminectomy but not by straight curettes or Kerrison punches. To decompress the contralateral side, somewhat wider interspinous space for simultaneous inserting of an endoscope and an instrument in the narrow midline space should be made with partial resection of the upper and lower ends of the spinous processes by a high speed burr (Fig. 6).

The above-mentioned strategies hopefully could lead us to safer and more successful BESS procedures for various lumbar spinal diseases.

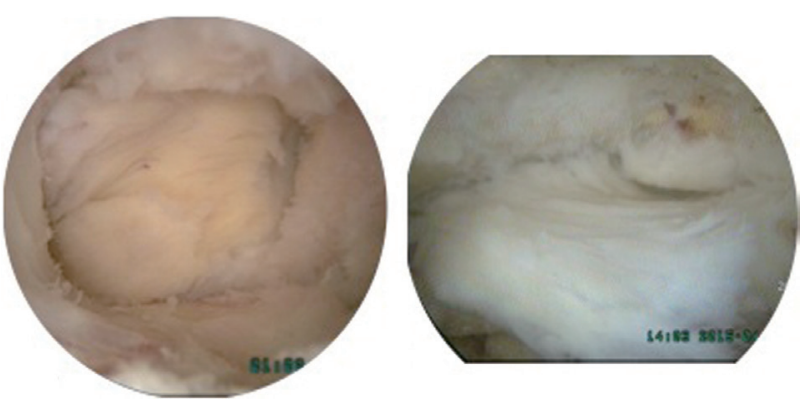

Fig. 6. Approach views from the left side. (A) An ipsilateral view on the left side after removing the outer layer of ligamentum flavum and laminectomy with a high-speed burr. (B) A contralateral view through the midline showed ligamentum flavum on the right side.

\section{Conclusions}

The BESS is newly tried technique in MISS for lumbar spine diseases and certain complications could be expected in an early learning period due to various operating conditions under fluid. The complications may more or less be avoided with more cases, allowing the surgeon to become accustomed to the surgical field. Fluent outflow of saline, control of epidural bleeding for a clear view, and successful flavectomy with angled curettes were the strategies in reducing complications with the new surgical technique.

\section{Conflict of Interest}

No potential conflict of interest relevant to this article was reported.

\section{References}

1. Polikandriotis JA, Hudak EM, Perry MW. Minimally invasive surgery through endoscopic laminotomy and foraminotomy for the treatment of lumbar spinal stenosis. J Orthop 2013;10:13-6.

2. Wong AP, Smith ZA, Lall RR, Bresnahan LE, Fessler RG. The microendoscopic decompression of lumbar stenosis: a review of the current literature and clinical results. Minim Invasive Surg 2012;2012:325095.

3. Birkenmaier C, Komp M, Leu HF, Wegener B, Ruetten S. The current state of endoscopic disc surgery: review of controlled studies comparing full-endoscopic procedures for disc herniations to standard procedures. Pain Physician 2013;16:335-44. 
4. Hu ZJ, Fang XQ, Zhou ZJ, Wang JY, Zhao FD, Fan SW. Effect and possible mechanism of musclesplitting approach on multifidus muscle injury and atrophy after posterior lumbar spine surgery. J Bone Joint Surg Am 2013;95:e192(1-9).

5. Tai CL, Hsieh PH, Chen WP, Chen LH, Chen WJ, Lai PL. Biomechanical comparison of lumbar spine instability between laminectomy and bilateral laminotomy for spinal stenosis syndrome: an experimental study in porcine model. BMC Musculoskelet Disord 2008;9:84.

6. Hartmann F, Janssen C, Bohm S, Hely H, Rommens PM, Gercek E. Biomechanical effect of graded minimal-invasive decompression procedures on lumbar spinal stability. Arch Orthop Trauma Surg 2012;132:1233-9.

7. Pao JL, Chen WC, Chen PQ. Clinical outcomes of microendoscopic decompressive laminotomy for degenerative lumbar spinal stenosis. Eur Spine J 2009;18:672-8.

8. Takaso M, Nakazawa T, Imura T, et al. Less invasive and less technically demanding decompressive procedure for lumbar spinal stenosis: appropriate for general orthopaedic surgeons? Int Orthop 2011;35:6773.

9. Mikami Y, Nagae M, Ikeda T, Tonomura H, Fujiwara $\mathrm{H}$, Kubo T. Tubular surgery with the assistance of endoscopic surgery via midline approach for lumbar spinal canal stenosis: a technical note. Eur Spine J 2013;22:2105-12.

10. Polikandriotis JA, Hudak EM, Perry MW. Minimally invasive surgery through endoscopic laminotomy and foraminotomy for the treatment of lumbar spinal stenosis. J Orthop 2013;10:13-6. 